J. Asiat. Soc. Bangladesh, Sci. 39(2): 139-146, December 2013

\title{
PHYTOCHEMICAL SCREENING OF SOME ANTIDYSENTERIC MEDICINAL PLANTS OF BANGLADESH
}

\author{
A. N. M. ALAMGIR ${ }^{1}$ AND KANIZ FATEMA \\ Department of Botany, University of Chittagong, Chittagong-4331, Bangladesh
}

\begin{abstract}
In this report, 40 antidysenteric medicinal plant species representing 24 families were considered for qualitative assessment of their secondary metabolites like alkaloids, flavonoids, glycosides, sterols and tannins. Alkaloids were present in all plant species, though in different degrees and the relative effectiveness of Dragendorffs' reagent was better than others. Distribution of flavonoids, glycosides, sterols and tannins was sporadic in different plant species except A. cepa, A. marmelos, I. coccinea, M. indica, S. dulcis and Z. officinale, where all these metabolites were present. Abundance and mode of distribution of secondary metabolites in different test plants and their organs were discussed.
\end{abstract}

Key words: Antidysenteric medicinal plants, Therapeutic principles, Secondary metabolites

\section{Introduction}

The use of medicinal plants has been a central component of health care in many cultures for centuries. The first recorded culturally significant plant residues of about 60000 years old were found in Iraq in 1960 at Neanderthal human burial site (Solecki and Shanidar 1975). About 30000 to 70000 plants are currently used by $80 \%$ of the rural people across the world for primary health care and WHO upholds quality, recommends and encourages the use of herbal drugs because of their easy availability, efficacy and, specially cost effectiveness compared to modern allopathic drugs (WHO 2002). More than 1000 plant species of Bangladesh are considered to have medicinal properties and about 455-747 have been described with their therapeutic uses for different diseases including dysentery (Mia 1990, Ghani 2003 and Yousuf et al. 2009).

Herbs which help in curing dysentery are antidysenteric and antidysenteric plants contain some active chemical agents, usually secondary metabolites, which function as therapeutic principles against dysentery. Dysentery is an inflammatory disorder of the lower intestinal tract, usually caused by microbial infection and resulting pain or fever or bloody diarrhoea. Dysenteric disease has long been recognized as a leading cause of morbidity and mortality among children (1-4 years) and aged people ( $\geq 60$ years) in Bangladesh (Mitra et al. 1990).

Medicinal plants produce a diverse assortment of secondary metabolites of therapeutic importance (Croteau et al. 2000 and Terryn et al. 2006) and plants with antidysenteric and antidiarrhoeal properties were found to contain alkaloids, flavonoids, saponins,

\footnotetext{
${ }^{1}$ Corresponding author: Email: alamgiranm@yahoo.com
} 
sterols, tannins and reducing sugars as well as show antibacterial and antiprotozoan activity (Longanga et al. 2000). However, phytochemical characteristics of many of the antidysenteric medicinal plants of Bangladesh are unknown. This paper deals with the phytochemical screening of antidysenteric medicinal plants available in the hilly area of Chittagong University campus and around.

\section{Materials and Methods}

During this investigation, 40 antidysenteric medicinal plant species, enlisted in different published literature (Ghani 2003 and Yousuf et al. 2009) were collected from the hilly area of Chittagong University campus and around covering different life forms such as herbs, shrubs and trees. Secondary metabolites like alkaloids, flavonoids, glycosides, sterols and tannins were analyzed qualitatively in the whole plant and plant parts within 6 h of collection. For alkaloids, a modified method (Amarasingham et al. 1965 and Apline and Cannon 1971) was followed. Dragendorff (D), Wagner (W), Mayer (M), Hager (H) and Tannic acid (T) were prepared following standard method (Cromwell 1955) and were used for alkaloid detection in $2 \% \mathrm{HCl}$ extract of the plant. Ethanol extract was used for the detection of flavonoids (Wall et al. 1954 and Farnsworth 1964). Sterols (Bhattacharjee and Das 1969), tannins (Wall et al. 1954) and glycosides (Eyjolfesson 1970) were assessed following the reported methods. The absence, presence and abundance of different secondary metabolites in test samples were indicated by,-+ and multiple of + signs, respectively. Each test was replicated thrice. The results are presented in Tables 1 and 2.

\section{Results and Discussion}

Qualitative analysis of alkaloids, flavonoids, glycosides, sterols and tannins of 40 antidysenteric medicinal plant species and/or their organs, e.g., root-rhizome, stem, bark, leaf, flower and fruit were carried out. From Table 1, it is apparent that alkaloids were present in all plant species and the extracts from various sources showed different responses to Dragendorff (D), Wagner (W), Mayer (M), Hager (H) and Tannic acid (T) reagents for alkaloids. Out of 225 tests by different reagents for alkaloids, 207 tests were positive indicating the presence of alkaloids in range of slight to abundant $(1+$ to $4+)$ and the number of positive response to reagents $\mathrm{D}, \mathrm{W}, \mathrm{M}, \mathrm{H}$ and $\mathrm{T}$ were $45,44,39,38$ and 41 , respectively. Negative response (-) in 18 tests indicated the inefficiency of the reagent used in the test. On the basis of alkaloid detecting efficacies, the relative effectiveness of the reagents may be graded as: $\mathrm{D}>\mathrm{W}>\mathrm{T}>\mathrm{M}>\mathrm{H}$. While evaluating 102 plant species of 47 families, Pasha (1977) reported positive response for alkaloids in 48 plant species only. On the other hand, positive response for alkaloids in 32 out of 42 plant species was reported by Tariq et al. (1987). In the present work, the degrees of responses $(1+$ to $4+)$ of 40 plant species and their parts to different alkaloid detecting reagents were different but a large number of tests were appeared to be strong positive (3+ to $4+)$. Kapoor et al. 
Phytochemical screening of some antidysenteric

Table 1. Qualitative analysis of alkaloids in 40 antidysenteric medicinal plants.

\begin{tabular}{|c|c|c|c|c|c|c|c|}
\hline \multirow{3}{*}{ Plant species } & \multirow{3}{*}{ Family } & \multirow{3}{*}{$\begin{array}{l}\text { Plant part } \\
\text { used }\end{array}$} & \multirow{2}{*}{\multicolumn{5}{|c|}{$\begin{array}{c}\text { Present }+ \text { / absent - abundant } \\
\mathrm{n}+ \\
\text { Reagents used }\end{array}$}} \\
\hline & & & & & & & \\
\hline & & & $\mathrm{D}$ & $\mathrm{W}$ & $\mathrm{M}$ & $\mathrm{H}$ & $\mathrm{T}$ \\
\hline Allium cepa & Liliaceae & bulb & + & + & + & + & + \\
\hline Allium sativum & Liliaceae & bulb & $3+$ & $3+$ & $2+$ & $2+$ & $2+$ \\
\hline Alstonia scholaris & Apocynaceae & bark & $2+$ & $3+$ & $3+$ & $2+$ & $3+$ \\
\hline $\begin{array}{l}\text { Andrographis } \\
\text { paniculata }\end{array}$ & Acanthaceae & leaf & $3+$ & $2+$ & $2+$ & $2+$ & $4+$ \\
\hline Aegle marmelos & Rutaceae & fruit & $3+$ & $2+$ & + & - & + \\
\hline $\begin{array}{l}\text { Ageratum } \\
\text { conyzoides }\end{array}$ & Asteraceae & leaf & $2+$ & - & - & - & - \\
\hline $\begin{array}{l}\text { Anacardium } \\
\text { occidentale }\end{array}$ & Anacardiaceae & bark & + & + & + & + & + \\
\hline $\begin{array}{l}\text { Barringtonia } \\
\text { acutangula }\end{array}$ & Barringtoniaceae & leaf & $4+$ & $4+$ & $2+$ & $2+$ & $2+$ \\
\hline $\begin{array}{l}\text { Calotrotropis } \\
\text { gigantea }\end{array}$ & Asclepiadaceae & root & + & $3+$ & + & + & + \\
\hline Calotropis procera & Asclepiadaceae & root & + & $3+$ & + & + & + \\
\hline $\begin{array}{l}\text { Catharanthus } \\
\text { roseus }\end{array}$ & Apocynaceae & leaf & $2+$ & $3+$ & - & - & $2+$ \\
\hline Cassia fistula & Caesalpiniaceae & stem bark & $2+$ & + & - & - & - \\
\hline Centella asiatica & Apiaceae & whole plant & $2+$ & + & + & + & $4+$ \\
\hline Cicer arietinum & Fabaceae & seed & $3+$ & $3+$ & $3+$ & $3+$ & $3+$ \\
\hline Cleome viscosa & Capparidaceae & leaf & $3+$ & + & $3+$ & $2+$ & $4+$ \\
\hline Cuminum cyminum & Apiaceae & seed & $3+$ & + & $2+$ & $2+$ & $2+$ \\
\hline \multirow{2}{*}{ Cocos nucifera } & \multirow{2}{*}{ Arecaceae } & flower & + & + & + & + & + \\
\hline & & kernel & + & + & + & + & + \\
\hline Curcuma longa & Zingiberaceae & rhizome & $2+$ & $2+$ & + & + & + \\
\hline Daucas carota & Apiaceae & rhizome & $2+$ & + & + & + & $2+$ \\
\hline Dalbergia sissoo & Fabaceae & leaf & + & + & $2+$ & $2+$ & + \\
\hline $\begin{array}{l}\text { Eupatorium } \\
\text { odoratum }\end{array}$ & Asteraceae & leaf & $3+$ & $2+$ & $2+$ & $2+$ & $3+$ \\
\hline \multirow{3}{*}{ Euphorbia hirta } & \multirow{3}{*}{ Euphorbiaceae } & leaf & $4+$ & + & + & $2+$ & $2+$ \\
\hline & & stem & $3+$ & + & - & + & + \\
\hline & & flower & $4+$ & + & + & $2+$ & $2+$ \\
\hline Ficus hispida & Moraceae & leaf & $2+$ & $2+$ & $2+$ & $3+$ & + \\
\hline \multirow{2}{*}{$\begin{array}{l}\text { Holarhena } \\
\text { antidysenterica }\end{array}$} & \multirow{2}{*}{ Apocynaceae } & leaf & $3+$ & + & $2+$ & $2+$ & + \\
\hline & & bark & $4+$ & $4+$ & $3+$ & $3+$ & + \\
\hline $\begin{array}{l}\text { Hibiscus rosa- } \\
\text { sinensis }\end{array}$ & Malvaceae & flower & $2+$ & + & $2+$ & + & $2+$ \\
\hline Ixora coccinea & Rubiaceae & flower & $2+$ & $2+$ & + & $2+$ & $2+$ \\
\hline $\begin{array}{l}\text { Jatropa } \\
\text { gossypifolia }\end{array}$ & Euphorbiaceae & leaf & + & + & + & + & + \\
\hline
\end{tabular}


(Contd.)

\begin{tabular}{|c|c|c|c|c|c|c|c|}
\hline Kalanchoe pinnata & Crassulaceae & leaf & $2+$ & + & + & + & $2+$ \\
\hline $\begin{array}{l}\text { Melastoma } \\
\text { malabathricum }\end{array}$ & Melasomaceae & leaf & $3+$ & + & - & - & - \\
\hline Mimosa pudica & Mimosaceae & root & + & + & + & + & + \\
\hline Mikania cordata & Asteraceae & leaf & $3+$ & $2+$ & $2+$ & $2+$ & $3+$ \\
\hline Morinda citrifolia & Rubiaceae & fruit & $2+$ & + & + & + & $4+$ \\
\hline Murraya koenigii & Rutaceae & leaf & $4+$ & $2+$ & $2+$ & $2+$ & $3+$ \\
\hline Mangifera indica & Anacardiaceae & bark & $4+$ & $3+$ & $3+$ & $3+$ & $4+$ \\
\hline Ocimum sp & Lamiaceae & leaf & + & + & + & + & + \\
\hline $\begin{array}{l}\text { Rauvolfia } \\
\text { serpentina }\end{array}$ & Apocynaceae & leaf & $3+$ & $3+$ & $2+$ & $3+$ & $3+$ \\
\hline Scoparia dulcis & Scrophulariaceae & leaf & $4+$ & $3+$ & $3+$ & $3+$ & $4+$ \\
\hline \multirow{2}{*}{ Solanum nigrum } & \multirow{2}{*}{ Solanaceae } & leaf & + & + & + & + & + \\
\hline & & fruit & + & + & - & - & - \\
\hline Tridax procumbens & Asteraceae & leaf & $3+$ & $3+$ & $3+$ & $3+$ & $3+$ \\
\hline Zinziber officinale & Zingiberaceae & rhizome & $2+$ & + & + & + & $2+$ \\
\hline
\end{tabular}

(1969) noted weak positive response for alkaloids while others (Pasha1977, Affandi et al. 2004) observed strong positive reactions ( $3+$ to $4+$ ) for alkaloids in a few plant species. The relative abundance of alkaloids found in the present work was higher in leaf, bark and rhizome than other organs of the test plants. With some minor exceptions leaf, stem and root of different medicinal plants were found to contain a broad spectrum of secondary metabolites including alkaloids, flavonoids, saponins etc. (Viji and Murugesan 2010, Pascaline et al. 2011). It appears that the distribution of alkaloids is uneven and sporadic within and among different antidysenteric medicinal plants of the present work. This finding supports the previous report (Chhetri et al. 2008) of a phytochemical screening for alkaloids and other bioactive chemicals.

Table 2. Qualitative analysis of flavonoids, glycosides, sterols and tannins in 40 antidysenteric medicinal plants.

\begin{tabular}{|c|c|c|c|c|c|c|}
\hline \multirow[t]{2}{*}{ Plant species } & \multirow[t]{2}{*}{ Family } & \multirow{2}{*}{$\begin{array}{l}\text { Plant } \\
\text { part } \\
\text { used }\end{array}$} & \multicolumn{4}{|c|}{ Secondary metabolites: + present /-absent } \\
\hline & & & Flavonoids & Glycosides & Sterols & Tannins \\
\hline Allium cepa & Liliaceae & bulb & + & + & + & + \\
\hline $\begin{array}{l}\text { Allium } \\
\text { sativum }\end{array}$ & Liliaceae & bulb & + & + & - & + \\
\hline $\begin{array}{l}\text { Alstonia } \\
\text { scholaris }\end{array}$ & Apocynaceae & bark & - & + & + & - \\
\hline $\begin{array}{l}\text { Andrographi } \\
\text { s paniculata }\end{array}$ & Acanthaceae & leaf & + & + & + & - \\
\hline $\begin{array}{l}\text { Aegle } \\
\text { marmelos }\end{array}$ & Rutaceae & fruit & + & + & + & + \\
\hline $\begin{array}{l}\text { Ageratum } \\
\text { conyzoides }\end{array}$ & Asteraceae & leaf & + & - & + & - \\
\hline
\end{tabular}


Phytochemical screening of some antidysenteric

(Contd.)

\begin{tabular}{|c|c|c|c|c|c|c|}
\hline $\begin{array}{l}\text { Anacardium } \\
\text { occidentale }\end{array}$ & Anacardiaceae & bark & - & - & + & - \\
\hline $\begin{array}{l}\text { Barringtonia } \\
\text { acutangula }\end{array}$ & Barringtoniaceae & leaf & + & - & + & + \\
\hline $\begin{array}{l}\text { Calotrotropis } \\
\text { gigantea }\end{array}$ & Asclepiadaceae & leaf & - & + & + & - \\
\hline $\begin{array}{l}\text { Calotropis } \\
\text { procera }\end{array}$ & Asclepiadaceae & leaf & - & + & + & - \\
\hline $\begin{array}{l}\text { Catharanthus } \\
\text { roseus }\end{array}$ & Apocynaceae & leaf & - & + & + & - \\
\hline $\begin{array}{l}\text { Cassia } \\
\text { fistula }\end{array}$ & Caesalpiniaceae & $\begin{array}{l}\text { stem } \\
\text { bark }\end{array}$ & + & + & - & - \\
\hline $\begin{array}{l}\text { Centella } \\
\text { asiatica }\end{array}$ & Apiaceae & $\begin{array}{l}\text { whole } \\
\text { plant }\end{array}$ & - & + & + & + \\
\hline $\begin{array}{l}\text { Cicer } \\
\text { arietinum }\end{array}$ & Fabaceae & seed & + & + & - & - \\
\hline $\begin{array}{l}\text { Cleome } \\
\text { viscosa }\end{array}$ & Capparidaceae & leaf & - & - & + & - \\
\hline $\begin{array}{l}\text { Cuminum } \\
\text { cyminum }\end{array}$ & Apiaceae & seed & + & + & - & - \\
\hline $\begin{array}{l}\text { Cocos } \\
\text { nucifera }\end{array}$ & Arecaceae & kernel & + & - & + & - \\
\hline $\begin{array}{l}\text { Curcuma } \\
\text { longa }\end{array}$ & Zingiberaceae & $\begin{array}{l}\text { rhizo } \\
\text { me }\end{array}$ & + & + & - & + \\
\hline $\begin{array}{l}\text { Daucas } \\
\text { carota }\end{array}$ & Apiaceae & $\begin{array}{l}\text { rhizo } \\
\text { me }\end{array}$ & - & - & - & + \\
\hline $\begin{array}{l}\text { Dalbergia } \\
\text { sissoo }\end{array}$ & Fabaceae & leaf & + & + & - & + \\
\hline $\begin{array}{l}\text { Eupatorium } \\
\text { odoratum }\end{array}$ & Asteraceae & leaf & + & - & - & - \\
\hline $\begin{array}{l}\text { Euphorbia } \\
\text { hirta }\end{array}$ & Euphorbiaceae & leaf & + & + & - & + \\
\hline $\begin{array}{l}\text { Ficus } \\
\text { hispida }\end{array}$ & Moraceae & leaf & - & + & - & + \\
\hline $\begin{array}{l}\text { Holarhena } \\
\text { antidysenter } \\
\text { ica }\end{array}$ & Apocynaceae & leaf & + & - & + & + \\
\hline $\begin{array}{l}\text { Hibiscus } \\
\text { rosa- } \\
\text { sinensis }\end{array}$ & Malvaceae & flower & + & - & - & + \\
\hline $\begin{array}{l}\text { Ixora } \\
\text { coccinea }\end{array}$ & Rubiaceae & flower & + & + & + & + \\
\hline $\begin{array}{l}\text { Jatropa } \\
\text { gossypifolia }\end{array}$ & Euphorbiaceae & leaf & + & - & - & + \\
\hline $\begin{array}{l}\text { Kalanchoe } \\
\text { pinnata }\end{array}$ & Crassulaceae & leaf & + & - & - & - \\
\hline
\end{tabular}




\begin{tabular}{|c|c|c|c|c|c|c|}
\hline $\begin{array}{l}\text { Melastoma } \\
\text { malabathric } \\
\text { um }\end{array}$ & Melasomaceae & flower & - & - & - & - \\
\hline $\begin{array}{l}\text { Mimosa } \\
\text { pudica }\end{array}$ & Mimosaceae & root & - & - & - & + \\
\hline $\begin{array}{l}\text { Mikania } \\
\text { cordata }\end{array}$ & Asteraceae & leaf & + & - & + & - \\
\hline $\begin{array}{l}\text { Morinda } \\
\text { citrifolia }\end{array}$ & Rubiaceae & leaf & - & + & + & + \\
\hline $\begin{array}{l}\text { Murraya } \\
\text { koenigii }\end{array}$ & Rutaceae & leaf & - & + & + & - \\
\hline $\begin{array}{l}\text { Mangifera } \\
\text { indica }\end{array}$ & Anacardiaceae & bark & + & + & + & + \\
\hline Ocimum $s p$ & Lamiaceae & leaf & - & + & + & - \\
\hline $\begin{array}{l}\text { Rauvolfia } \\
\text { serpentina }\end{array}$ & Apocynaceae & leaf & - & - & + & - \\
\hline $\begin{array}{l}\text { Scoparia } \\
\text { dulcis }\end{array}$ & $\begin{array}{l}\text { Scrophulariace } \\
\text { ae }\end{array}$ & leaf & + & + & + & + \\
\hline $\begin{array}{l}\text { Solanum } \\
\text { nigrum }\end{array}$ & Solanaceae & leaf & - & + & + & - \\
\hline $\begin{array}{l}\text { Tridax } \\
\text { procumbens }\end{array}$ & Asteraceae & leaf & + & - & + & - \\
\hline $\begin{array}{l}\text { Zinziber } \\
\text { officinale }\end{array}$ & Zingiberaceae & $\begin{array}{l}\text { rhizo } \\
\text { me }\end{array}$ & + & + & + & + \\
\hline
\end{tabular}

Results presented in Table 2 for 4 other metabolites (e.g., flavonoids, glycosides, sterols and tannins) show that all except the flower of $M$. malabathricum gave positive response to tests for one or more metabolites. Out of the total 160 tests, about 92 tests were positive. Among the lot, 6 plant species or plant parts (e.g., A. cepa, A. marmelos, I. coccinea, $M$. indica, $S$. dulcis and $Z$. officinale) gave positive response for all 4 metabolites (e.g., flavonoids, glycosides, sterols and tannins) while 9, 17 and 7 plant species or plant parts gave positive responses for 3, 2 and 1 metabolite, respectively. For each of flavonoids and glycosides, 24 plant species gave positive response whereas for sterols and tannins positive responses were in 25 and 19 species, respectively. It appears that the distribution of flavonoids, glycosides and sterols in the test plants and their parts was comparatively wider than that of tannin but all showed sporadic and uneven distribution in different plant species and their parts. Tariq et al. (1987) in his work with the members of Asteraceae, noted positive responses for flavonoids, sterols, tannins and saponins in 21, 22, 20 and 4 plant species, respectively. In the present work, 6 plant species, e.g., A. cepa, A. marmelos, I. coccinea, M. indica, S. dulcis and Z. officinale contained all 5 secondary metabolites but in the rest of the plants their distribution is uneven. The presence of alkaloids, flavonoids, glycosides and steroids in Citrullus seeds has been reported (Ambil et al. 2007). Ayoola et al. (2008) reported on the presence of flavonoids, terpenoids, saponins, tannins and reducing sugars in Carica papaya, 
Magnifera indica, Psidium guajava, and Vernonia amygdalina has been found. Cardiac glycosides and alkaloids were absent in $M$. indica while alkaloids and phenolic compounds, anthraquinones, were absent in P. guajava and $V$. amygdalina, respectively. Sivasankari et al. (2010) while examining the major metabolites like carbohydrates, tannins, saponins, flavonoids, alkaloids, betacyanins, quinones, terepenoids, phenols, glycosides and cardiac glycosides in Caesalpinia pulcherrima (a domesticated shrub) and Caesalpinia bonduc (a wild shrub) leaf extracts reported their uneven distribution in the plant species and the wild plants contributed high values for the secondary metabolites than the domesticated. A wide range of secondary metabolites were reported to be present in different antidysenteric, antidiarrhoeal and other medicinal plants (Longanga et al. 2000, Satyanarayana and Eswaraiah 2010 and Narayanasamy and Ragavan 2012). Therefore, the secondary metabolites identified in different medicinal plants of the present work may be considered as active therapeutic agents against the dysenteric disease.

\section{References}

Affandi, H., A. Nuryadin and S. B. Prayogo. 2004. Medicinal herbs of Pasir Mayang, Jambi: Ethnopharmacy and toxicity screening. Biotropia. 22: $40-58$.

Amarasingham, R. D., N. G. Bisset, A. H. Millard and M. C. Woods.1965. A phytochemical survey of Malaya part III. Alkaloids and saponins. Econ. Bot. 18: 290-295.

Ambil, A. A, E. M. Abdurrahman, M. I. Sule, U. U. Pateh, Y. R. Abdurrahman and D. G. Ibrahim. 2007. Phytochemical screening and histopathological studies of Colosynthis citrullus in albinprats. Nig. Journ. Pharm. Sci. 6 (2):7 - 13.

Apline, T. E. H. and J. R.Canon.1971. Distribution of alkaloids in some Western Australian plants. Econ. Bot. 25(4):366-380.

Ayoola, G. A., H. A. B.Coker, S. A. Adesegun, A. A. Adepoju-Bello, K. Obaweya, E. C. Ezennia and T. O. Atangbyila. 2008. Phytochemical screening and antioxidant activities of some selected medicinal plants used for malaria therapy in Southwestern Nigeria. Tropic. J. Pharm. Res. 7(3): 1019-1024.

Bhattacharjee, A. K. and A. K. Das.1969. Phytochemical screening of few Mysore plants. Econ. Bot. 23(3):204-206.

Chhetri, H. P., N. S.Yogol, J. Sherchan, K. C. Anupa, S. Mansoor and P. Thara. 2008. Phytochemical and antimicrobial evaluations of some medicinal plants of Nepal. Kathmandu Univ. J. Sci. Eng. \& Technol. I(V): 49-54.

Cromwell, B. T. 1955. Modern method of plant analysis. Springer - Veriag, Berlin. 373-374 pp.

Croteau, R., Toni M. Kutchan and Norman G. Lewis. 2000. Natural products (Secondary metabolites). In: Biochemistry \& Molecular Biology of Plants (eds. B. Buchanan, W. Gruissem, R. Jones). American Society of Plant Physiologists. 1250-1318pp.

Eyjolfesson, R.1970. Recent advances in the chemistry of cyanogenic glycosides. Frog.Org.Nat.Pro. 27:75-108.

Farnsworth, N.R.1964. Biological and phytochemical screening of plants. J. Pharm. Sci. 55(3): 225-276.

Ghani, A. 2003. Medicinal plants of Bangladesh with chemical constituents and uses. Asiatic Soc. Bangladesh, Dhaka. 66-434pp. 
Kapoor, L. D., A. Singh, S. L. Kapoor and S. N. Srivastava. 1969. Suvery of Indian plants for saponin, alkaloids and flavpnoids. Lioydia. 32(3): 397-303.

Longana Otshudi, A., A. Vercruysse and A. Foriers. 2000. Contribution to the ethnobotanical, phytochemical and pharmacological studies of traditionally used medicinal plants in the treatment of dysentery and diarrhoea in Lomela area, Democratic Republic of Congo (DRC). J. Ethnopharmacology. 71(3):411-423.

Mia, M. M. K. 1990. In: Traditional medicine. (ed. A. Ghani). Jahangirnagar University. Savar, Dhaka. 73pp.

Mitra, A. K., N. C. Engleberg, R. I. Glass and M. K. Chowdhury .1990. Fatal dysentery in rural Bangladesh. J. Diarrhoeal Dis Res. 8(1-2):12-17.

Narayanasamy, K. and B. Ragavan. 2012. Phytochemical and mineral screening of Zanthoxylum Tetraspermum (W \& A). E-J.Chem. 9(1), 121-130. http://www.e-journals.net

Pascaline, J., M. Charles, C. Lukhoba and O. George. 2011. Phytochemical constituents of some medicinal plants used by the Nandis of South Nandi district, Kenya. J. Animal \& Plant Scis. 9(3): 1201- 1210.

Pasha, M. K. 1977. Phytochemical survey of Bangladesh plants. I. A preliminary report. Bangladesh J. Bot. 6(1+2): $57-64$.

Sivasankari, K., S. Janaky and T. Sekar. 2010. Evaluation of phytochemicals in selected medicinal plants of the Caesalpinia species. Indian J. Sci. Technol. 3(12):1118-1121

Solecki, R. and I. V. Shanidar. 1975. A Neanderthal flower burial in northern Iraq. Science. 190: $880-881$.

Satyanarayana,T. and M. Chinna Eswaraiah.2010. In-vitro antioxidant and free radical scavenging potential of stem of Calycopteris floribunda. Res. J. Pharmaceutic., Biol. and Chem. Sci. 1:(2): 117-123.

Tariq, M., J. S. Mossa, M. A. Al-yahya, I. A . Al-meshal and A. A. Al-badar. 1987. Phytochemical and biological screening of Saudi medicinal plants Part-10. A study on Saudi plants of Family Compositae. Int. J. Crude Drug Res. 25(1): 17-25.

Terryn, N., M. Van Montagu, D. Inze and A. Goossens. 2006. Functional genomic approaches to study and engineer secondary metabolism in plant cell cultures.In: Medicinal and Aromatic Plants. (eds.R.J. Bogers, L.E. Craker and D. Lange.). Springer. Printed in the Netherlands. 291-300pp.

Viji, M. and S. Murugesan. 2010. Phytochemical analysis and antibacterial activity of medicinal plant Cardiospermum halicacabum Linn. .J. Phytol. 2(1): 68-77.

Wall, M . E., M . M . Krider, C. F. Krewson, C. R. Eddy, J. J. Willaman, S. S. Corell and H. S. Gentry. 1954. Steroidal sapogenins. Survey of plants for steroidal sapogenins and other constituents. J Pharm. 1-7.

WHO. 2002. Traditional medicines strategy. WHO 2002-2005, World Health Organization, Geneva. Switzerland. 43-49pp.

Yusuf, M.,J. U. Chowdhury, M. N. Hoque and J. Begum. 2009.Medicinal plants of Bangladesh. BCSRI, Chittagong-4220. Bangladesh. 1-692 pp.

(Received revised manuscript on 10 October 2013) 